\title{
Quantitative Electroencephalography and Exploding Head Syndrome: A Case Study
}

\author{
Elmar Weiler', Rainer Wiegand', Klaus Brill', Dieter Schneider²
}

\begin{abstract}
We describe the case of a 65 year old female patient suffering from the exploding head syndrome (EHS). The characteristics of EHS is the perception of sudden loud noises while falling asleep and or during the period of waking up. The syndrome does not cause any pains, however, the medical condition of the female subject is characterized by fear, distress and sleeping problems. Comparison of qEEG data pre and post EHS event revealed significant alterations in the EEG patterns.
\end{abstract}

\section{Keywords}

Exploding head syndrome, Quantitative electroencephalography (Qeeg)

\section{Introduction}

In 1920 Armstrong-Jones [1] described EHS as a "snapping sound in the brain", which lacked any objective external acoustic stimulation [1]. The term "exploding head syndrome" (EHS) was first coined by Pearce [2] in 1989. It is described as a benign disorder occurring in a relaxed state and or at the transition of sleep-wake or wake-sleep. The first case of EHS was published by Mitchell [3] as early as 1876 . He reported a case where the patient suffered from a nocturnal event described as 'pistol shot' [3]. In a later publication he used the term 'sensory shock' and recognised it as a sleep disorder [4]. The onset of EHS is mainly observed in older individuals. Thus far there have been no published data clearly indicating risk factors for EHS. An elaborate review of the EHS - related literature revealed that there is a 1.55: 1 female to male predominance [5].

The course of EHS appears to be fairly variable, with patients reporting a single episode in a lifetime to many episodes per night [4]. The course of EHS can be constant or irregular, and may go into a full or partial remission only to be followed by a seemingly unexpected reoccurrence of attacks. Affected subjects are suffering from fear, shock and surprise. In about $10 \%-20 \%$ of these patients the occurrence of "flashing lights" were reported. Furthermore episodes of tachycardia and palpitations are described as common features [4]. Consequently a sleeping disorder might evolve. A variety of studies revealed a correlation between a disturbed sleep and poor health outcomes, including falls [6], medical morbidity [7], psychiatric symptoms [8], and cognitive impairment [9].

The pathogenetic mechanism of EHS remains to be elucidated. Thus far five possible mechanisms have been discussed

1. A variety of ear dysfunctions have been assumed.

2. Brief temporal lobe complex partial seizures have been considered as well [9].

\footnotetext{
${ }^{1}$ NeuroNet, -Neurofeedback Therapie Zentrum-, Parkstrasse 23, D-6606 St. Wendel, Germany

${ }^{2}$ Klinik und Poliklinik für Hals-, Nasen- und Ohrenkrankheiten, plastische und ästhetische Operationen Universität Würzburg Haus B2 Josef-Schneider-Str. 11 D-97080 Würzburg, Germany

${ }^{\dagger}$ Author for correspondences: Elmar Weiler, NeuroNet, -Neurofeedback Therapie Zentrum-, Parkstrasse 23, D-6606 St. Wendel, Germany, Tel: 0049(0)685193330, email: e.weiler@neuronet.de; neuronet2009@web.de
} 
3. Ehs might result from as a possible side-effect of rapid withdrawal from either benzodiazepines or selective serotonin reuptake inhibitors. [10].

4. Most recently a transient dysfunction of the calcium channel was correlated to ehs [10-12].

5. The final model assumes a dysfunctional neuronal network during the transition from wakefulness to sleep located within the brainstem [13-16].

We present the case of a female patient suffering from EHS.

\section{Materials and Methods}

\section{- Patient}

A 65 - year-old right - handed female patient, suffering from the exploding head syndrome over the last sixteen years, was examined in our institute. The EHS occurs every day during transition of awake to sleep, thus leading to a sleeping disorder. A signed informed consent was obtained along with a detailed medical history. The patient then underwent a brainwave exam and the data were subjected to a computerassisted EEG analysis.

\section{- Quantitative Electroencephalography}

The recording of the electroencephalographic activity was carried out with a Neurosearch 24 instrument Lexicor Medical Technology, Inc., Boulder, CO). Nineteen electrodes were positioned on the scalp in accordance with the standard international 10-20 system. The brainwaves were collected with 19 electrodes. The following electrode sites were employed: Fp1, Fp2, F7, F3, Fz, F4, F8, T3, C3, Cz, C4, T4, T5, P3, Pz, P4, T6, O1, O2, and referenced to linked ears. The female subject was seated in an arm chair and with eyes closed in a sound-attenuated, electrically shielded room. Brainwaves were recorded at rest and after the occurrence of EHS.

The collected data were visually inspected, epochs containing artefacts e.g. eye movements, drowsiness, coughing, speaking, were not included in the data analysis.

The screened artefact-free data were subsequently analysed. Numerical representation of the calculated data and a presentation of the data in form of power spectra were realised. Statistical calculations were performed using only artefact - screened data. The data were calculated for the following frequency bands: delta $(2,0-4,0 \mathrm{~Hz})$, theta $(4,5-8,0 \mathrm{~Hz})$, alpha $(8,5-13 \mathrm{~Hz})$, alpha $_{1}(7,0-9,0 \mathrm{~Hz})$, alpha $2(9,0$ $-11,0 \mathrm{~Hz})$, alpha $3(11,0-13,0 \mathrm{~Hz})$ and beta (13,5-21.0 Hz).

\section{- Pittsburgh Sleep Quality Index (PSQI)}

The PSQI is an internationally known, reliable questionnaire (19 self-rated questions and 5 questions rated by a bed partner) for evaluating sleep quality. In retrospective, for a period of four weeks, the assessment of sleep quality, sleep latency, sleep duration, the frequency of sleep disturbing events, the use of sleep medications, sleep efficiency as well as the daytime tiredness are evaluated. A total of 19 items are used for quantitative evaluation and assigned to 7 components. Each item can assume a value range of 0 to 3 meaning 0 (no difficulty) to 3 (severe difficulty). The total score can range from 0 to 21 . A total score greater than 5 allows the classification into poor sleep [17].

\section{Results}

\section{Pittsburgh Sleep Quality Index (PSQI)}

Table 1 demonstrates the distribution of the different subscales of the Pittsburgh Sleep Quality Index. The highest score 3 was noted for sleep latency and use of sleep medications, however, a score of 0 was determined the component daytime dysfunctions a score 0 . In general a total score $>5$, is correlated with a significant disturbance in sleep quality. In our case a total score of 11 was noted.

\section{- Average Total Power}

The calculated average total power under baseline condition with eyes open was $30.4 \pm 44,1 \mu \mathrm{V}^{2}$; a significant decrease of the average total power $27.2 \pm 31.1 \mu \mathrm{V}^{2} .(\mathrm{p}=0,003)$ was calculated for the period post EHS.

For baseline condition with eyes closed the calculated average total power was $60.0 \pm 123.6$ $\mu \mathrm{V}^{2}$. An increase of the average total power $66.0 \pm 103.6 \mu \mathrm{V}^{2}$ following the EHS event was calculated, however, barely missing significance $(p=0.06)$.

\section{Calculation of Total Average Power for the Delta, Theta, Alpha, and Beta Frequency Bands}

Average total power was calculated for the following four frequency bands: delta (2.0-4.0 $\mathrm{Hz})$, theta $(4.5-8.0 \mathrm{~Hz})$, alpha $(8.5-13.0 \mathrm{~Hz})$ 
, and beta $(13.5-21.0 \mathrm{~Hz})$ under the condition eyes closed and eyes open pre and post EHS event. The EEG data recorded after the EHS event with the eyes closed exhibit a significant increase of the total average power for the slow frequency bands (delta, theta) and for the fast frequency band (beta) as compared to the pre EHS recorded EEG data. No significant change was determined for the alpha waves (Table 2A).

The EEG data recorded after the EHS event with the eyes open revealed a significant decrease of the total average power for the alpha and beta bands as compared to the pre EHS recorded EEG data. No significant changes were calculated for the delta and theta bands (Table 2B).

Alpha waves were subdivided into alpha ${ }_{1}$, alpha and alpha A $_{\text {. }}$ A significant increase of power for the alpha ${ }_{1}$ band and a significant decrease of power for the alpha ${ }_{3}$ band and no significant change for the alpha ${ }_{2}$ band (Table 3A) under the condition eyes closed when comparing pre and post EHS EEG data.

Comparison of the quantitative EEG data pre vs post EHS (condition eyes open) revealed a significant decrease for two alpha frequency bands namely, alpha ${ }_{2}$ and alpha $_{3}$ (Table 3B). No significant changes were determined for the alpha frequency band (Table 3B).

Furthermore a significant decrease in power for the $12 \mathrm{~Hz}$ frequency band was noted under the condition eyes closed and eyes open when comparing EEG data recorded pre and post EHS (Table 4A and Table 4B) in the temporoparieto-occipital region.

\section{Discussion}

Exploding head syndrome (EHS) appears to be an unusual, non-life threatening event that can manifest during any part of the sleep cycle. The characteristic of this syndrome is a sudden unexpected loud noise/bang resembling an explosion. Sometimes the appearance of flashes of lights is being described. The concomitants of these events are feelings of fear or anxiousness with tachycardia and sweating. The attacks demonstrate no consistency in respect to frequency. Attacks can be a daily as well as a one-time event. The event can reoccur with alternating frequencies and even being absent for longer periods of time $[1,4]$.

Several publications describe EHS as a harmless condition, because no pathological

$\begin{aligned} & \text { Table 1: Presentation of the results for the seven PSQI items and the PSQI } \\
& \text { global score. }\end{aligned}$
\begin{tabular}{|l|l|}
\hline PSQI component (scale range) & Score \\
\hline 1. subjective sleep quality [0-3] & $\mathbf{2}$ \\
\hline 2. Sleep latency [0-3] & $\mathbf{3}$ \\
\hline 3. Sleep duration [0-3] & $\mathbf{1}$ \\
\hline 4. Habitual sleep efficiency [0-3] & $\mathbf{1}$ \\
\hline 5. Sleep disturbances [0-3] & $\mathbf{1}$ \\
\hline 6. Use of sleep medication [0-3] & $\mathbf{3}$ \\
\hline 7. Daytime dysfunction [0-3] & $\mathbf{0}$ \\
\hline PSQI global score (0-21) & $\mathbf{1 1}$ \\
\hline
\end{tabular}

Table 2 A: Comparison of total average power of frequency bands during rest and following exploding head syndrome episode with eyes closed.

\begin{tabular}{|l|l|l|l|l|}
\hline Eyes closed & Delta $\left(\boldsymbol{\mu} \mathbf{V}^{2}\right)$ & Theta $\left(\boldsymbol{\mu} \mathbf{V}^{2}\right)$ & Alpha $\left(\boldsymbol{\mu} \mathbf{V}^{2}\right)$ & Beta $\left(\boldsymbol{\mu} \mathbf{V}^{2}\right)$ \\
\hline baseline & $18.73 \pm 17.06$ & $42,55 \pm 51.55$ & $152.54 \pm 214.69$ & $26.07 \pm 19.59$ \\
\hline post EHS episode & $27.35 \pm 28.79$ & $55.01 \pm 78.32$ & $151.98 \pm 158.60$ & $29.28 \pm 21.33$ \\
\hline $\boldsymbol{p}$-value & $<0.0001$ & 0.0003 & ns & 0.003 \\
\hline
\end{tabular}

Table 2B: Comparison of total average power of frequency bands during rest and following exploding head syndrome episode with eyes open (B).

\begin{tabular}{|l|l|l|l|l|}
\hline Eyes open & Delta $\left(\boldsymbol{\mu} \mathbf{V}^{2}\right)$ & Theta $\left(\boldsymbol{\mu} \mathbf{V}^{2}\right)$ & Alpha $\left(\boldsymbol{\mu} \mathbf{V}^{2}\right)$ & Beta $\left(\boldsymbol{\mu} \mathbf{V}^{2}\right)$ \\
\hline baseline & $20.14 \pm 24.81$ & $25.02 \pm 41.72$ & $54.91 \pm 66.00$ & $21.41 \pm 15.45$ \\
\hline post EHS episode & $19.43 \pm 23.56$ & $27.55 \pm 32.86$ & $42.73 \pm 40.57$ & $19.44 \pm 15.43$ \\
\hline $\boldsymbol{p}$-value & ns & ns & $<0.0001$ & 0.02 \\
\hline
\end{tabular}

Table 3A: Comparison of total average power of alpha frequency bands during rest and following exploding head syndrome episode with eyes closed (A).

\begin{tabular}{|l|l|l|l|}
\hline Eyes closed & Alpha $_{1}\left(\boldsymbol{\mu} \mathbf{V}^{2}\right)$ & Alpha $_{\mathbf{2}}\left(\boldsymbol{\mu} \mathbf{V}^{2}\right)$ & Alpha $_{\mathbf{3}}\left(\boldsymbol{\mu} \mathbf{V}^{\mathbf{2}}\right)$ \\
\hline baseline & $65.10 \pm 120.25$ & $107.69 \pm 170.77$ & $39.11 \pm 49.85$ \\
\hline post EHS episode & $87.83 \pm 114.92$ & $117.53 \pm 137.08$ & $24.31 \pm 26.35$ \\
\hline p-value & 0.0003 & ns & $<0.0001$ \\
\hline
\end{tabular}

Table 3B: Comparison of total average power of alpha frequency bands during rest and following exploding head syndrome episode with eyes open.

\begin{tabular}{|l|l|l|l|}
\hline Eyes open & Alpha $_{1}\left(\boldsymbol{\mu} \mathbf{V}^{\mathbf{2}}\right)$ & Alpha $_{\mathbf{2}}\left(\boldsymbol{\mu} \mathbf{V}^{\mathbf{2}}\right)$ & Alpha $_{\mathbf{3}}\left(\boldsymbol{\mu} \mathbf{V}^{2}\right)$ \\
\hline baseline & $30.80 \pm 67.51$ & $30.94 \pm 38.61$ & $20.62 \pm 22.56$ \\
\hline post EHS episode & $30.10 \pm 40.63$ & $24.61 \pm 31.15$ & $14.30 \pm 12.25$ \\
\hline p-value & Ns & 0.0007 & $<0.0001$ \\
\hline
\end{tabular}

Table 4A: Calculation of the percent change of average power for the 12$\mathrm{Hz}$ band under the condition eyes closed.

\begin{tabular}{|l|l|l|l|}
\hline Eyes closed & Pre EHS & post EHS & Percentage \\
\hline Electrode Site & $\mathbf{1 2 - H z}$ Band $\left(\boldsymbol{\mu \mathbf { V } ^ { 2 } )}\right.$ & $\mathbf{1 2 - H z ~ B a n d ~}\left(\boldsymbol{\mu \mathbf { V } ^ { 2 } )}\right.$ & Change \\
\hline T4 & $22.16 \pm 19.53$ & $14.09 \pm 13.82$ & $\mathbf{- 3 6}$ \\
\hline T5 & $35.86 \pm 24.66$ & $18.28 \pm 12.90$ & $-\mathbf{4 9}$ \\
\hline P3 & $57.81 \pm 43.57$ & $30.88 \pm 21.73$ & $\mathbf{- 4 7}$ \\
\hline PZ & $59.26 \pm 47.39$ & $33.19 \pm 24.80$ & $\mathbf{- 4 4}$ \\
\hline P4 & $80.37 \pm 59.82$ & $40.44 \pm 28.18$ & $\mathbf{- 5 0}$ \\
\hline T6 & $70.49 \pm 69.38$ & $36.16 \pm 24.13$ & $\mathbf{- 4 9}$ \\
\hline $\mathbf{O 1}$ & $97.44 \pm 79.69$ & $50.25 \pm 39.69$ & $\mathbf{- 4 8}$ \\
\hline $\mathbf{O 2}$ & $108.19 \pm 95.43$ & $64.03 \pm 46.44$ & $\mathbf{- 4 1}$ \\
\hline
\end{tabular}




Table 4B: Calculation of the percent change of average power for the 12-
Hz band under the condition eyes open.
\begin{tabular}{|l|l|l|l|}
\hline Eyes open & Pre EHS & post EHS & \% change \\
\hline Electrode Site & $\mathbf{1 2 - H z}$ Band $\left(\boldsymbol{\mu \mathbf { V } ^ { 2 } )}\right.$ & $\mathbf{1 2 - H z}$ Band $\left(\boldsymbol{\mu} \mathbf{V}^{2}\right)$ & \\
\hline T4 & $16.00 \pm 13.24$ & $10.43 \pm 8.85$ & $-\mathbf{3 5}$ \\
\hline T5 & $18.93 \pm 17.54$ & $15.77 \pm 11.98$ & $-\mathbf{1 7}$ \\
\hline P3 & $26.83 \pm 24.57$ & $18.80 \pm 14.00$ & $\mathbf{- 3 0}$ \\
\hline PZ & $28.37 \pm 22.73$ & $15.83 \pm 12.80$ & $\mathbf{- 4 4}$ \\
\hline P4 & $33.44 \pm 25.68$ & $17.54 \pm 13.05$ & $\mathbf{- 4 8}$ \\
\hline T6 & $28.71 \pm 28.46$ & $16.54 \pm 15.60$ & $\mathbf{- 4 2}$ \\
\hline $\mathbf{O 1}$ & $30.78 \pm 35.90$ & $21.86 \pm 18.74$ & $\mathbf{- 2 9}$ \\
\hline $\mathbf{O 2}$ & $35.76 \pm 33.40$ & $18.40 \pm 15.01$ & $\mathbf{- 4 9}$ \\
\hline
\end{tabular}

electroencephalographic activities were detected, neurological disorder never developed, and the symptoms always ameliorated over time. As to our knowledge this is the first report describing changes of EEG patterns when comparing EEG tracings obtained pre and post the EHS event.

Under the condition eyes closed an increase of power for the delta, theta and beta band following the EHS event was noted, however, no changes were detected for the alpha power. It is tempting to speculate whether the increase of the power for the delta waves and theta waves post EHS might correlate with the intensity of sound and with emotional distress. Similar results were reported for tinnitus patients $[18,19]$.

An increase of power for a $16 \mathrm{~Hz}$ band was detected in a tinnitus patient [20]. In contrast to the from tinnitus suffering patient the EHS affected subject exhibited a significant decline in power for the $12 \mathrm{~Hz}$ brain wave mainly observed in the temporo-parietal region of the brain. Mazaheri \& Picton [21] described a decrease of alpha in the parietal region of the brain upon response to auditory targets. The underlying mechanisms for this response remain thus far elusive. A possible explanation of the described decrease in alpha within the parietal region of the brain might be an increased excitability of the auditory network and/or the parietal region of the brain. The alpha decrease due to an auditory stimulus might indicate high-level processes [18]. The brain structures responsible for the decrease of alpha in response to auditory stimulus remain to be determined. However, we can still propose that these structures are engaged in the processing of the novel sounds.

EHS is not life threatening, and treatment is generally limited to reassurance and stress management. Reassurance reduces anxiety. In some patients EHS is experienced as very disturbing and thus leading to severe sleep problems. In these cases, patients benefited from the use of appropriate medication. The drug therapy was found to either alleviate or resolve EHS related symptoms [11-13]. If stress triggers exploding head symptoms, then some form of relaxation should be employed. Stress relief could include short walks, reading before bed and yoga. In our case, we successfully employed neurofeedback therapy. This approach led to an improved sleeping profile associated with a decline in EHS episodes. These initial observations need further validation.

\section{References}

1. Armstrong-Jones R. Snapping of the brain. Lancet 196(1), 720 (1920).

2. Pearce JM. Clinical features of the exploding head syndrome. J. Neurol. Neurosurg. Psychiatry 52(7), 907-910 (1989).

3. Mitchell SW. On some of the disorders of sleep. Virginia. Med. Monthly 11(1), 769-781 (1876).

4. Mitchell SW. Some disorders of sleep. Am. J. Med. Sci 100(1), 109-127 (1890).

5. Sharpless BA. Exploding head syndrome. Sleep. Med. Rev 18(1), 489-493 (2014).

6. Stone KL, Ewing SK, Lui LY, et al. Self-reported sleep and nap habits and risk of falls and fractures in older women: the study of osteoporotic fractures. J. Am. Geriatr. Soc 54(8), 1177-1183 (2006).

7. Gottlieb DJ, Redline S, Nieto FJ, et al. Association of usual sleep duration with hypertension: the Sleep Heart Health Study. Sleep 29(8), 1009-1014 (2006).

8. Friedman L, Brooks JO, Bliwise DL, et al. Insomnia in older adults: Relations to depression and anxiety. American Journal of Geriatric Psychiatry: Am. Psychiatric. Assn 17(6), 153-159 (1993).

9. Blackwell T, Yaffe K, Ancoli-Israel S, et al. Poor Sleep Is Associated With Impaired Cognitive Function in Older Women: The Study of Osteoporotic Fractures. J. Gerontol. A. Biol. Sci. Med. Sci 61(4), 405-410 (2006).

10. Ganguly G, Mridha B, Khan A, et al. Exploding head syndrome: a case report. Case. Rep. Neurol 5(1), 14-17 (2013).

11. Jacome DE. Exploding head syndrome and idiopathic stabbing headache relieved by nifedipine. Cephalalgia 21(5), 617-618 (2001).

12. Palikh GM, Vaughn BV. Topiramate responsive exploding head syndrome. J. Clin. Sleep.
Med 15(6), 382-383 (2010).

13. Chakravarty A. Exploding head syndrome: report of two new cases. Cephalalgia 28(4), 399-400 (2007).

14. Evans RW, Pearce JM. Exploding head syndrome. Headache 41(1), 602-603 (2001).

15. Cohen AS, Kaube H. Rare nocturnal headaches. Curr. Opin. Neurol 17(3), 295-299 (2004).

16. Queiroz LP. Unusual headache syndromes. Headache 53(1), 12-22 (2013).

17. Buysse DJ, Reynolds CF III, Monk TH, et al. Pittsburgh Sleep Quality Index. [PSQI] In Handbook of Psychiatric Measures: $2^{\text {nd }}$ Edtn. American Psychiatric Publishing, Washington DC (2008).

18. Weiler EWJ, Brill K, Tachiki KH, et al. Neurofeedback and Quantitative Electroencephalography. Int. Tinnitus. J 8(2), 87-93 (2002).

19. Brill K, Weiler, EWJ. Dorntherapy: Its Effect 


\section{Quantitative Electroencephalography and Exploding Head Syndrome: A Case Study Research}

on Electroencephalographic Activity in

Tinnitus Patients with Craniocervical Dys-

function. Int. Tinnitus. J 9(2), 138-142 (2003).
20. Weiler EWJ, Brill K, Tachik KH. Quantitative Electroencephalography and Tinnitus: A Case Study. Int. Tinnitus. J 6(2), 124-126 (2000).
21. Mazaheri A, Picton TW. EEG spectral dynamics during discrimination of auditory and visual targets. Brain. Res. Cogn. Brain. Res 24(1), 81-96 (2005) 